\title{
Total laparoscopic extirpation of a fixed uterus from benign gynecological disease
}

\author{
M. Sami Walid • Richard L. Heaton
}

Received: 14 December 2009 / Accepted: 8 January 2010/Published online: 4 February 2010

(C) Springer-Verlag 2010

\begin{abstract}
Frequently, a fixed pelvis is encountered that is caused by a benign disease, either severe endometriosis or severe adhesions with or without fibroid uterus. We present two cases of nulligravida and multiparous women who had absolute frozen pelvises with no motion whatsoever of their pelvic structures on bimanual examination. Conventionally, these patients would have been approached by open hysterectomy only. We do not consider a frozen pelvis from what appears to be a benign case a contraindication to the laparoscopic approach.
\end{abstract}

Keywords Fixed pelvis $\cdot$ Impacted uterus $\cdot$ Retroverted uterus · Obliterated cul-de-sac · Adhesions · Endometriosis · Laparoscopic hysterectomy

\section{Introduction}

A fixed pelvis usually implies the possibility of malignancy spread along the broad ligaments and the parametrium to the pelvic sidewall. However, more frequently, a fixed pelvis is caused by a benign disease, either severe endometriosis or severe adhesions with the bilateral tuboovarian complexes adherent to the pelvic sidewalls or from a large fibroid uterus associated with scar tissue or endometriosis $[1,2]$.

\footnotetext{
M. S. Walid

Medical Center of Central Georgia,

840 Pine Street, Suite 880 ,

Macon, GA 31201, USA

e-mail: mswalid@yahoo.com

R. L. Heaton $(\bowtie)$

Heart of Georgia Women's Center,

209 Green Street,

Warner Robins, GA 31099, USA

e-mail: riclheaton@yahoo.com
}

The following two cases are of women who had absolute frozen pelvises with no motion whatsoever of their pelvic structures on bimanual examination. Conventionally, these patients have been approached by open hysterectomy and considered unfit for laparoscopic treatment [3]. This has not been our experience. We do not consider the frozen pelvis in what appears to be a benign case a contraindication to the laparoscopic approach.

\section{Case 1}

A 42-year-old woman, gravida 0 , was admitted for the definitive surgical treatment of her chronic cyclic pelvic pain, dyspareunia, menorrhagia, and a large fibroid uterus. The patient had a fixed pelvis on pelvic exam. On laparoscopy, she was found to have dense adhesions with an obliterated posterior cul-de-sac with the uterus fixed in place and immobile. Total laparoscopic hysterectomy, bilateral salpingo-oophorectomy, ureterolysis, and enterolysis were performed. The uterus weighed $208 \mathrm{~g}$. Operative time was $131 \mathrm{~min}$, and estimated blood loss was $150 \mathrm{~cm}^{3}$. Figures 1, 2, and 3 are from this case.

\section{Case 2}

A 39-year-old woman, gravida 3 para 3, complained of menometrorrhagia, chronic pelvic pain, and deep dyspareunia, worse on the left. On pelvic exam, her uterus was retroverted, and the right ovary was felt in the posterior cul-de-sac, which was suggestive of adhesions. Her left ovary was enlarged, filling the left half of the pelvic cavity. The uterus and adnexa felt fixed in place. Ultrasound revealed what appeared to be a hemorrhagic cyst in the right ovary and a large cystic mass in the left ovary that was single-locular and thin-walled, 


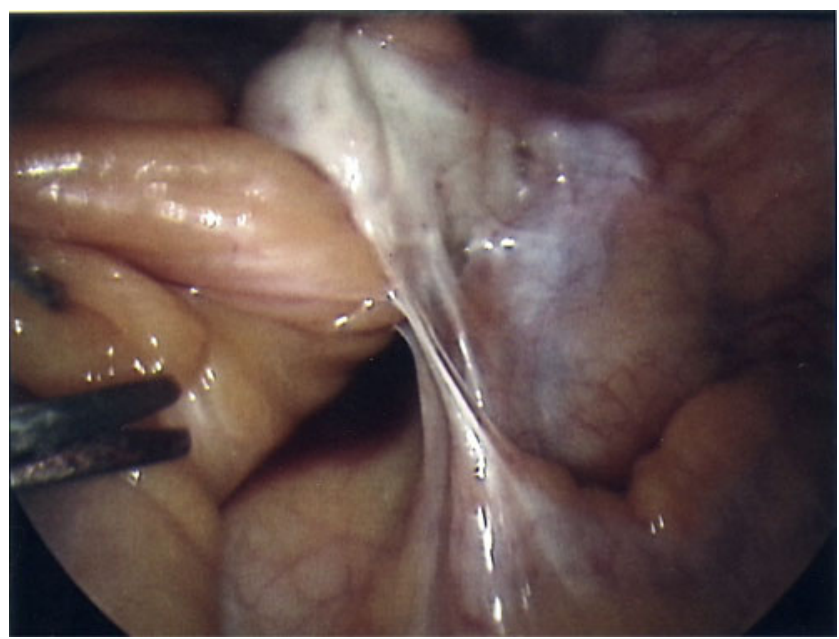

Fig. 1 Adhesions of the sigmoid to the left ovary (case 1)

measuring $7.8 \mathrm{~cm}$ in largest diameter. Her CA-125 was 47 (minimally elevated). She had a score of nine on Pelvic Pain and Urgency/Frequency questionnaire. Her findings were suggestive of endometriosis. There was no adenopathy on computed tomography scan but some dilatation of the left ureter, suggestive of partial obstruction and, most likely, some retroperitoneal fibrosis on the left. The patients had a history of three C-sections. She was counseled about her options and decided with the physician to proceed with laparoscopy with possible conversion to open surgery. The patient was found to have bilateral endometriomas, left larger than right; tubo-ovarian complexes scarred into the pelvic sidewall on both sides; dense adhesions of the vesicouterine reflection (from prior C-sections); and severe enteric adhesions. The patient underwent total laparoscopic hysterectomy, bilateral salpingo-oophorectomy, bilateral ureterolysis, and enterolysis. The operation lasted $147 \mathrm{~min}$. Blood loss was estimated at $100 \mathrm{~cm}^{3}$. On cystoscopy and hydrodistension, the patient was found to have glomerulations throughout the

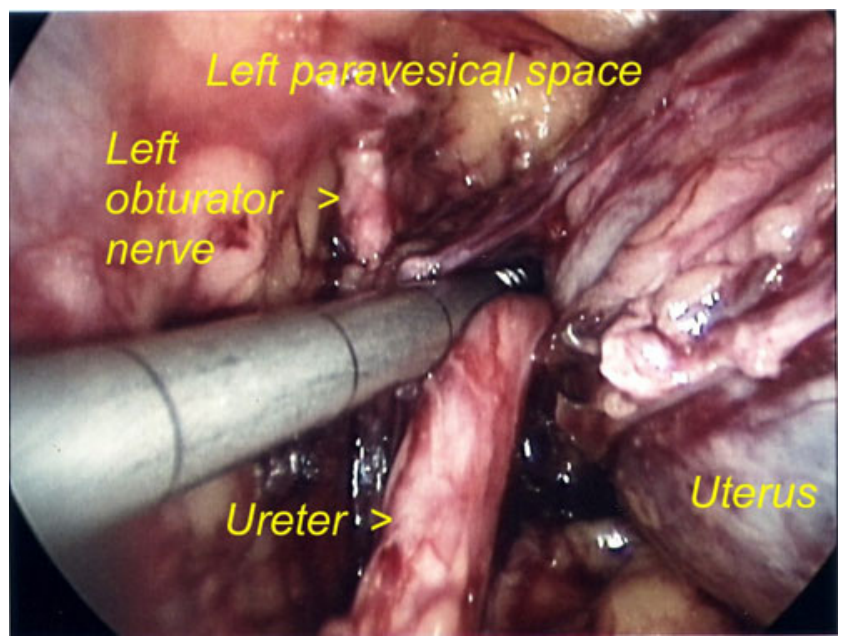

Fig. 2 Ureterolysis in process (case 1)

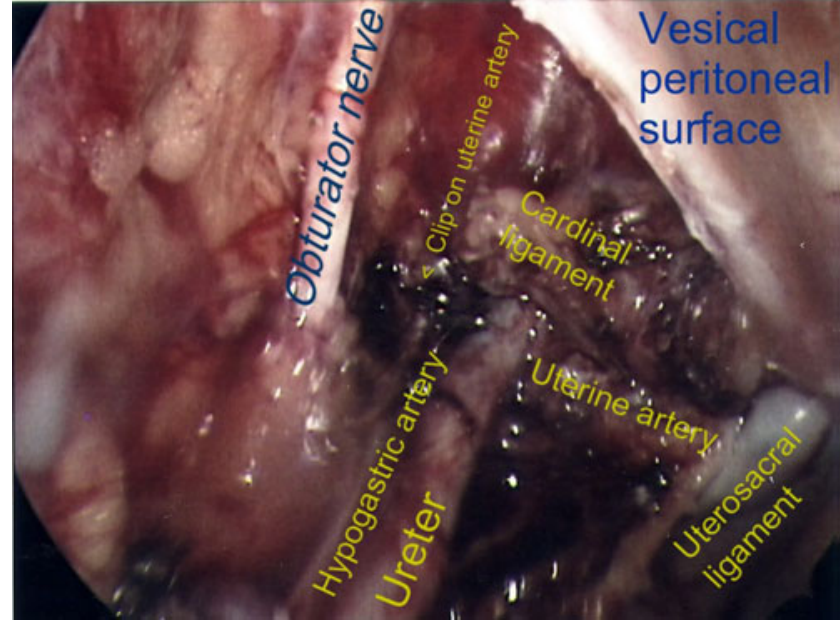

Fig. 3 Ureterolysis completed (case 1)

bladder, consistent with interstitial cystitis. The removed material weighed $231 \mathrm{~g}$. Operative time was $147 \mathrm{~min}(2 \mathrm{~h}$ and $27 \mathrm{~min}$ ), and estimated blood loss was $100 \mathrm{~cm}^{3}$. Figures 4, 5, and 6 are from this case.

\section{Discussion}

When dealing with cases of frozen pelvis, the surgeon should always follow the surgical principles of exposure of normal organs that should not be injured. You take what the individual case easily offers you in way of adhesiolysis in order to restore normal anatomy. You begin by deflecting the sigmoid colon, medially separating its attachment to the left pelvic sidewall and exposing the bifurcation of the external iliac artery and the internal iliac artery (the hypogastric artery). Normally, the left ureter will be found coursing over or close to this junction which allows one to begin the retroperitoneal pelvic dissection that allows these

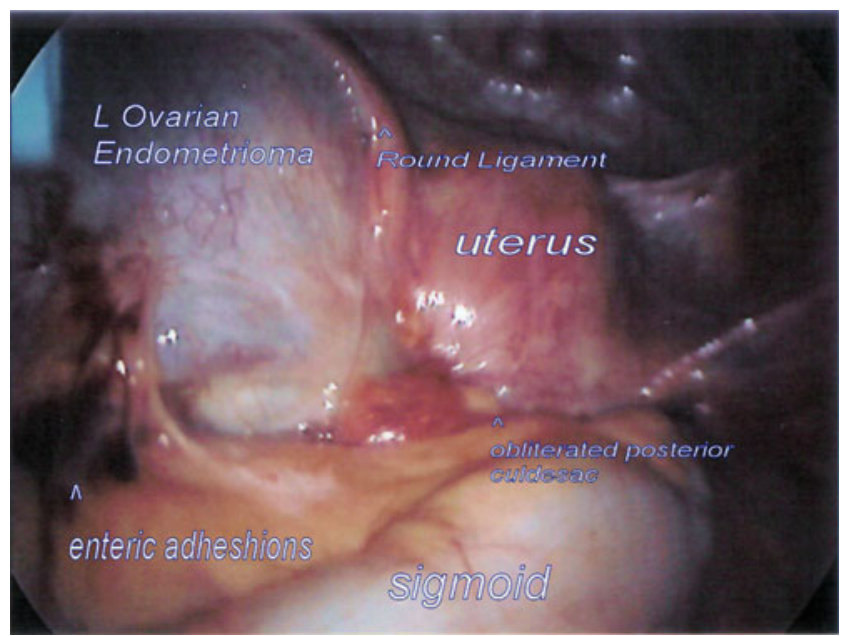

Fig. 4 Impacted fixed uterus (case 2) 


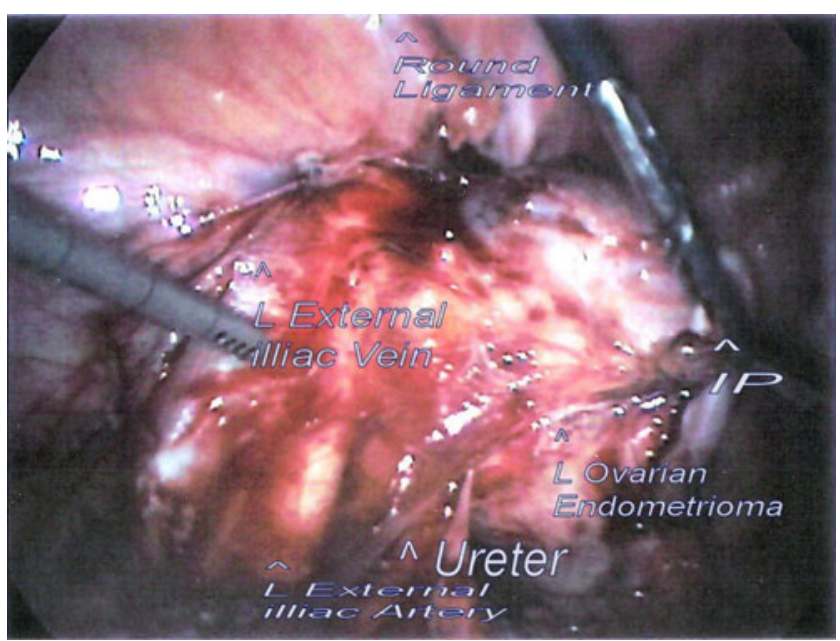

Fig. 5 Exposing the bifurcation of the iliac artery (case 2)

cases to be safely done. Initially, the dissection of the ureter is begun retroperitoneally. Once there is a safe margin between the infundibulopelvic (IP) ligament and the ureter, the IP is coagulated with bipolar or harmonic energy or tied up giving further anterior exposure and enabling deeper advancement of the ureterolysis procedure. Normally, at this point, the round ligament can also be coagulated (to control bleeding from Samson's artery) and divided, giving further exposure to the course of the ureter and allowing the uterus and adherent bowel to be displaced medially. At this point, the peritoneum of the anterior broad ligament can be taken down past the pelvic midline on the left side exposing the cervix and allowing further medial displacement of the adherent uterus and sigmoid. This allows optimal visualization of the pelvis on the left so the ureter can be easily followed to where it disappears into the cardinal ligament web. The uterine artery will reliably be found coming over the ureter at this level and, depending on the anatomy, can be clipped, tied, or bipolar-coagulated lateral to the ureter

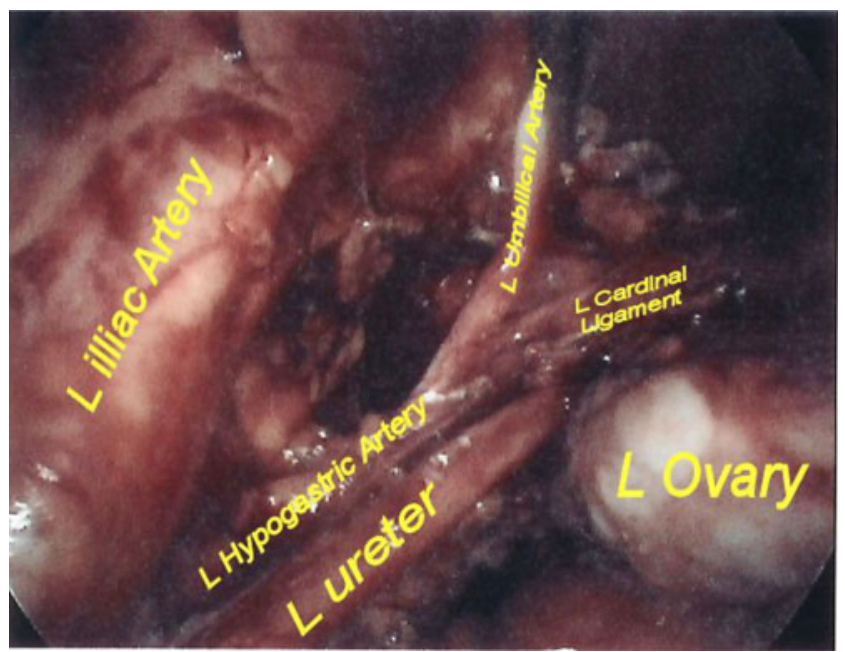

Fig. 6 The iliac bifurcation (case 2) controlling the third vascular pedicle coming to the uterus from the left side. At this point, the dissection is taken to the right side, and exactly the same procedure is followed. This results in near-complete devascularization of the uterus with exception of possible input from the descending cervical branch of the uterine artery if it has a collateral attachment to the azygos vaginal artery. At this point, if there are significant vesicouterine adhesions anteriorly from prior C-sections, infection, or endometriosis, these are taken down to further free the adherent adnexal-uterine-sigmoid mass. This is usually done easily by coming in under the scar bands laterally turning the active blade of the harmonic ace down into the cervix and pulling briskly through the edge of the scar band. This process can be serially repeated until the scar bands are completely separated from the cervix. The normal areolar plane between the bladder and the lower uterine segment can now be entered and the bladder taken down off of the lower uterine segment, cervix, and upper vagina. The dissection of the posterior cul-de-sac is then addressed. Medial retroperitoneal dissection is done utilizing the prior ureterolysis incisions. This allows visualization of the retroperitoneal course of the sigmoid allowing safer dissection of the sigmoid from the adnexal structures and lateral uterus. The final step of the dissection is then ready to be done. With the uterus devascularized, the serosal plane of the uterus can be dissected deliberately erring on the side of myometrial entry. Since the uterus is devascularized and bleeding is minimal, the easily recognized myometrium allows continual correction to the serosal plane and safe dissection of the sigmoid from the back of the uterus and reopening the posterior culde-sac. The patient can now have standard completion of the total laparoscopic hysterectomy.

These cases usually take at least $2-3 \mathrm{~h}$ for an experienced laparoscopist. They can be time-consuming but are usually not difficult to do as long as the principles set above are followed.

Conflict of interest There is no actual or potential conflict of interest in relation to this article.

\section{References}

1. Kataoka ML, Togashi K, Yamaoka T, Koyama T, Ueda H, Kobayashi H, Rahman M, Higuchi T, Fujii S (2005 Mar) Posterior cul-de-sac obliteration associated with endometriosis: MR imaging evaluation. Radiology 234(3):815-823

2. Amin-Hanjani S, Nyirjesy P (1994) Incarcerated fibroid uterus: a rare cause of acute abdominal pain in a nonpregnant patient. $\mathrm{J}$ Gynecol Surg 10(2):97-98

3. Agency for Health Care Policy and Research (1995) Treatment of common non-cancerous uterine conditions: issues for research. conference summary. AHCPR Publication No. 95-0067, July 1995. Agency for Health Care Policy and Research, Rockville. http://www.ahrq.gov/research/uterine.htm 